\title{
CORIOCARCINOMA MANIFESTANDO-SE INICIALMENTE COMO UM TUMOR CEREBRAL
}

\author{
José Alberto G. DA Silva * \\ Ely Chaves **
}

O coriocarcinoma (corioepitelioma) é uma neoplasia altamente maligna, originada do tecido coriônico e ocorrendo aproximadamente em cada 25.000 gravidezes ${ }^{5}$. Cêrca de $50 \%$ dêstes tumores são precedidos de mola hidatiforme, $25 \%$ seguem um abôrto, 22,5\% uma gravidez normal e uma pequena percentagem $(2,5 \%)$ se relaciona a gestação ectópica ${ }^{11}$. Além do coriocarcioma com a sua usual localização primária na cavidade uterina, existem numerosos exemplos desta neoplasia ocorrendo em situações ditas ectópicas como as observadas no mediastino ${ }^{21}$, ovários $4,8,15,18$, testículos $6,9,10$, bexiga $^{3}$. As metástases mais freqüentes são observadas nos pulmões, vagina, cérebro, rins e baço. Park e Less ${ }^{19}$, estudando cêrca de 295 casos de corioepitelioma com metástases, encontraram uma incidência de 53,59\% de metástases pulmonares, seguindo-se, em ordem decrescente, as metástases localizadas na vagina $(35,59 \%)$, rins $(11,52 \%)$, cérebro $(8,15 \%)$, baço $(8,13 \%)$, ovários $(5,42 \%)$. A grande freqüência das metástases pulmonares, vaginais e cerebrais têm sido confirmada por outros autores ${ }^{1,6,7,17}$.

$\mathrm{Na}$ presença de um coriocarcinoma intra-uterino, não há dificuldade em se detetar ou de se suspeitar da ocorrência de lesão cerebral metastástica, a qual pode se apresentar sob duas variedades: (1) como um tumor cerebral de evolução progressiva, com cefaléia, náuseas, vômitos, estase papilar, sonolência, associadas a outros sinais de hipertensão craniana; (2) tipo hemiplégico, o qual costuma se manifestar sùbitamente com um quadro clínico de ictus ou hemorragia cerebral com sintomas apopletiformes ${ }^{2}$.

No caso que passamos a descrever, a tumoração localizada no cérebro de uma jovem de 19 anos foi interpretada inicialmente como um tumor primário, do ponto de vista clínico. O diagnóstico histopatológico de coriocarcinoma levou a um estudo retrospectivo, resultando na descoberta de um coriocarcinoma intra-uterino, até então assintomático.

$$
\text { O B S E R V A C A O }
$$

M.G.F., 19 anos, branca, casada, internada em 2-7-1970 (Reg. 812, Hospital Santa Izabel). A paciente relatou que há aproximadamente 45 dias , teve forte

* Professor de Neurologia e Neurocirurgia na Fac. Med. Univ. Paraiba; ** Professor de Anatomia Patológica na Fac. Med. Univ. Paraíba e Chefe do Depart. Patologia, Hospital do Cancer Napoleão Laureano, Paraíba. 
cefaleia, com ulterior perda da consciência e impossibilidade de movimentar os membros direitos. O exame neurológico mostrou acentuada disfasia motora, paralisia facial central direita, edema de papila bilateral, mais acentuado à esquerda, hemiplegia à direita, predominando no membro superior, hipertomia elástica dos membros direitos com hiperreflexia; sinais de Hoffman, Babinski e Rossolino à direita. A angiografia carotidiana esquerda evidenciou um processo expansivo provocando desvio da artéria cerebral anterior para a direita, além de vasos patológicos na massa tumoral. Com o diagnóstico de tumor cerebral, a paciente foi submetida à craniotomia osteoplástica frontal esquerda para a exérese da tumoracão que ocupava a maior parte da porção posterior do lobo frontal esquerdo. Foi feita a ablação parcial do lobo citado, tendo sido ressecada, macroscòpicamente, tôda a massa tumoral. O exame histopatológico mostrou tratar-se de um coriocarcinoma. Com êste diagnóstico, voltamos a examinar a paciente dirigindo, desta feita, a anamnese em direção ao aparelho genital, tendo a paciente informado que há 4 meses dera à luz à uma criança normal, queixando-se apenas de discreta perda sanguínea pelos genitais. Um exame citológico da secreção cérvicovaginal revelou a presença de células epiteliais atipicas (classe V de Papanicolau). A paciente foi submetida à histerectomia total com anexectomia bilateral.

A paciente recebeu alta dois meses após a segunda intervenção, tendo o último contrôle neurológico revelado a regressāo da hemiplegia, persistindo, no entanto, discreta disfasia motora, além de perturbaçōes da esfera psiquica. Ulteriormente tivemos noticia do seu falecimento, ocorrido 4 meses após a intervençāo neurocirúrgica.

Exame anátomo-patológico do material colhido nas intervençôes cirúrgicas e nas vias e órgãos genitais - Nas porções provenientes do tumor cerebral, de consistência friável e coloração vermelho-acastanhada, os cortes mostram a presença de um tecido neoplásico maligno constituido por massas celulares, nas quais destacam-se citotrofoblastos (células de Langhans) e sinciciotrofoblastos em intima associaçāo. As referidas células infiltram o tecido cerebral em grande extensão (fıg. 1), acompanhado-se de infiltração inflamatória. Moderada proliferação de células histiocitárias (micróglia) de distribuição multifocal e exibindo citoplasma amplo, apresentando numerosos vacúolos e núcleos centrais e hipercromáticos (fig. 2). Estas células são melhor observadas nas proximidades das áreas de necrose e hemorragia. Nota-se, na substância branca, a presença de microcistos, além de distenção das bainhas de mielina (fig. 3). A maioria dos segmentos venosos encontra-se revestido por endotélio normal, porém, em algumas áreas, nota-se o envolvimento à maneira de manguito, dos segmentos venosos por células do tipo sincicial (fig. 4).

Os esfregaços vaginais, corados segundo a técnica de Papanicolau, mostram grande número de hemácias, leucócitos polimorfonucleares neutrófilos, células histiocitárias, além de moderada esfoliação de células superficais com predominio das células précornificadas. Chama a atenção a presença de células epiteliais atípicas nos esfregaços vaginais (fig. 5), exibindo citoplasma irregular, núcleo hipercromático e com cromatina irregularmente distribuida (fig. 6).

Utero de consistência amolecida. Canal endocervical permeável em tôda a extensão, recoberto por secreçăo sero-sanguinolenta. Ao nivel do fundus nota-se pequena formação nodular exibindo, ao córte, tecido vermelho-acastanhado. A referida tumoração é vista infiltrando a parêde miometrial, estando a serosa livre do comprometimento neoplásico. Os ovários mostram numerosas formações císticas as quais, ao exame microscópico, revelaram tratar-se de cistos luteinicos. Histològicamente, a tumoração intra-uterina revelou as mesmas características morfológicas da descrita no cérebro, sendo constituída por proliferação atípica de elementos trofoblásticos (cito e sinciciotrofoblastos) que infiltram a parêde miometrial (fig. 7). Densa infiltração linfo-monocitária, bem como numerosas áreas de hemorragia e necrose foram observadas. Em outras porções nota-se a predominância de células sinciciotrofoblásticas infiltrando o miométrio (fig. 8). Preparacōes obtidas mediante impressão direta da tumoração sôbre lâminas e, ulteriormente, coradas pelo método de 
Papanicolau, mostram numerosas células gigantes com núcleos arredondados ou ovais e hipercromáticos, citoplasma anfófilo. As células citotrofoblásticas apresentam-se em grupos compactos (fig. 9) ou isoladamente, exibindo núcleo arredondado ou oval, com cromatina grosseira e irregular e com macronucléolos acidófilos. O citoplasma destas células exibe macrovacúolos (fig. 10).
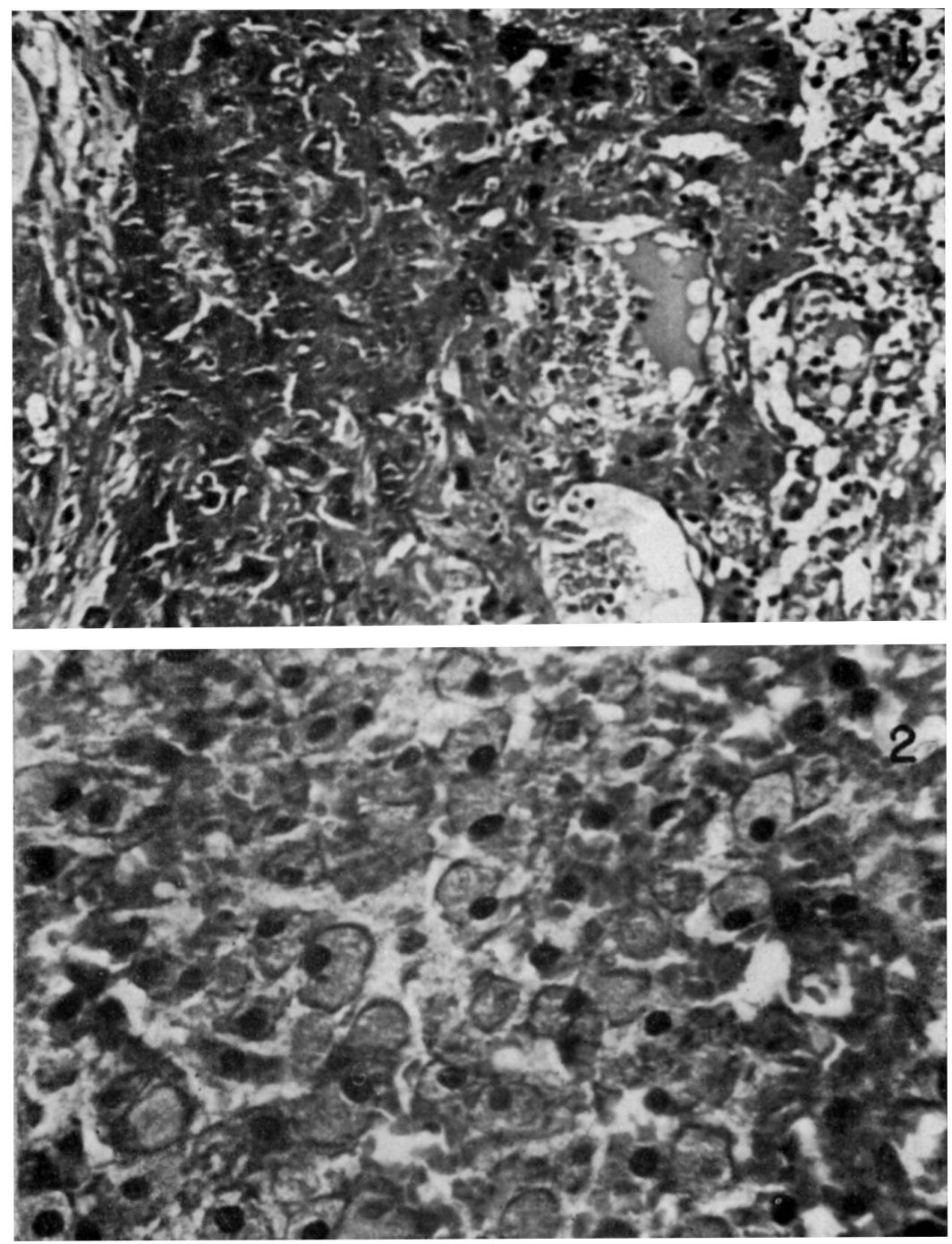

Fig. 1 - Caso M.G.F. Coriocarcinoma metastático no cérebro, mostrando massas de células citotrofoblásticas, envolvidas por sinciciotrofoblastos $(H . E ., 120 \times)$.

Fig. 2 - Proliferaçĩo histiocitica (microgliocitica) focal. (H.E., $1000 \times)$. 

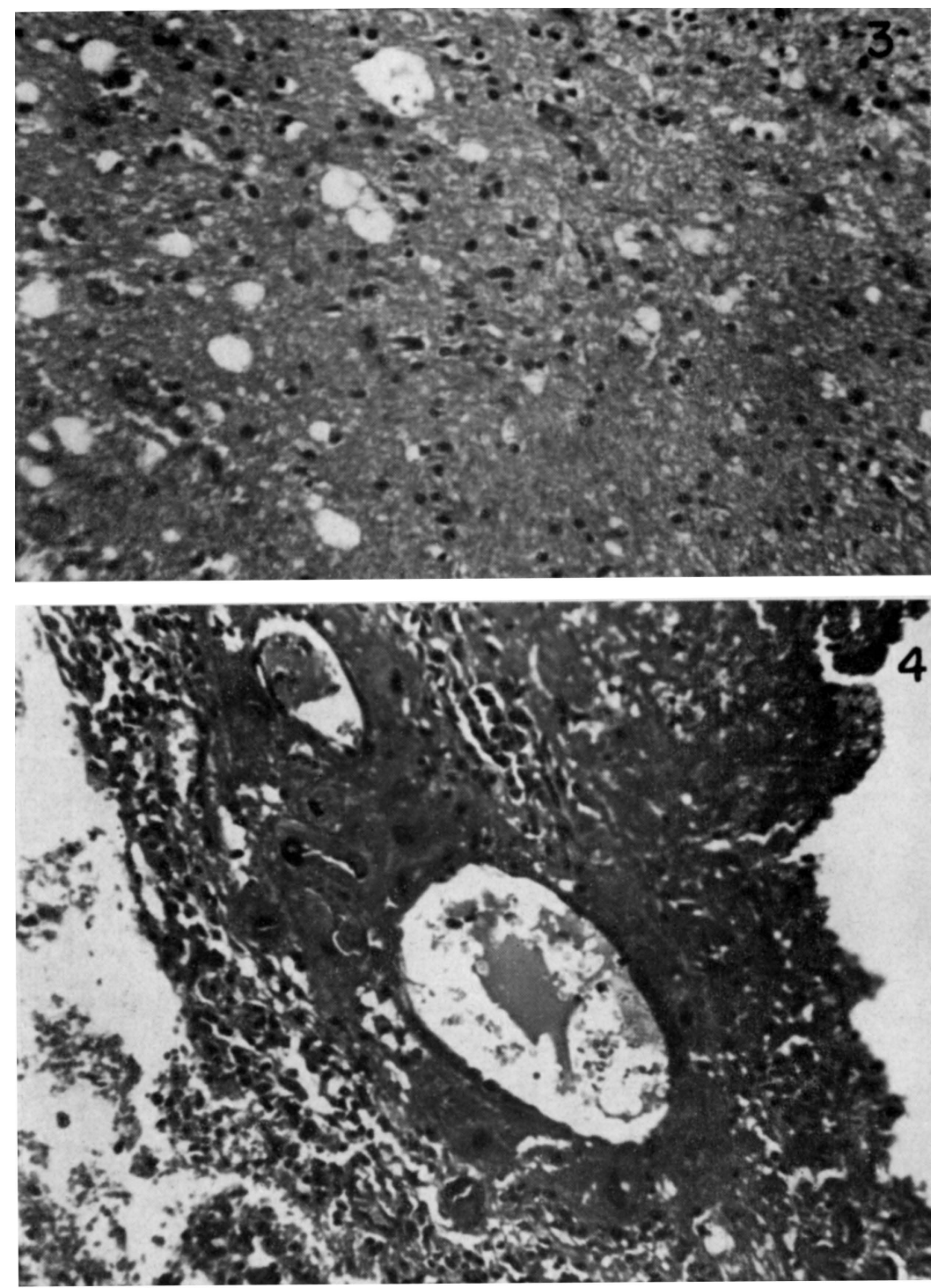

Fig. 3 - Substancia branca exibindo micro-vacuolização (H.E., $120 \times)$.

Fig. 4 - Células sinciciais e citotrofoblásticas dispondo-se em tôrno de um segmento venoso, no tecido cerebral (H.E., $450 \times)$. 

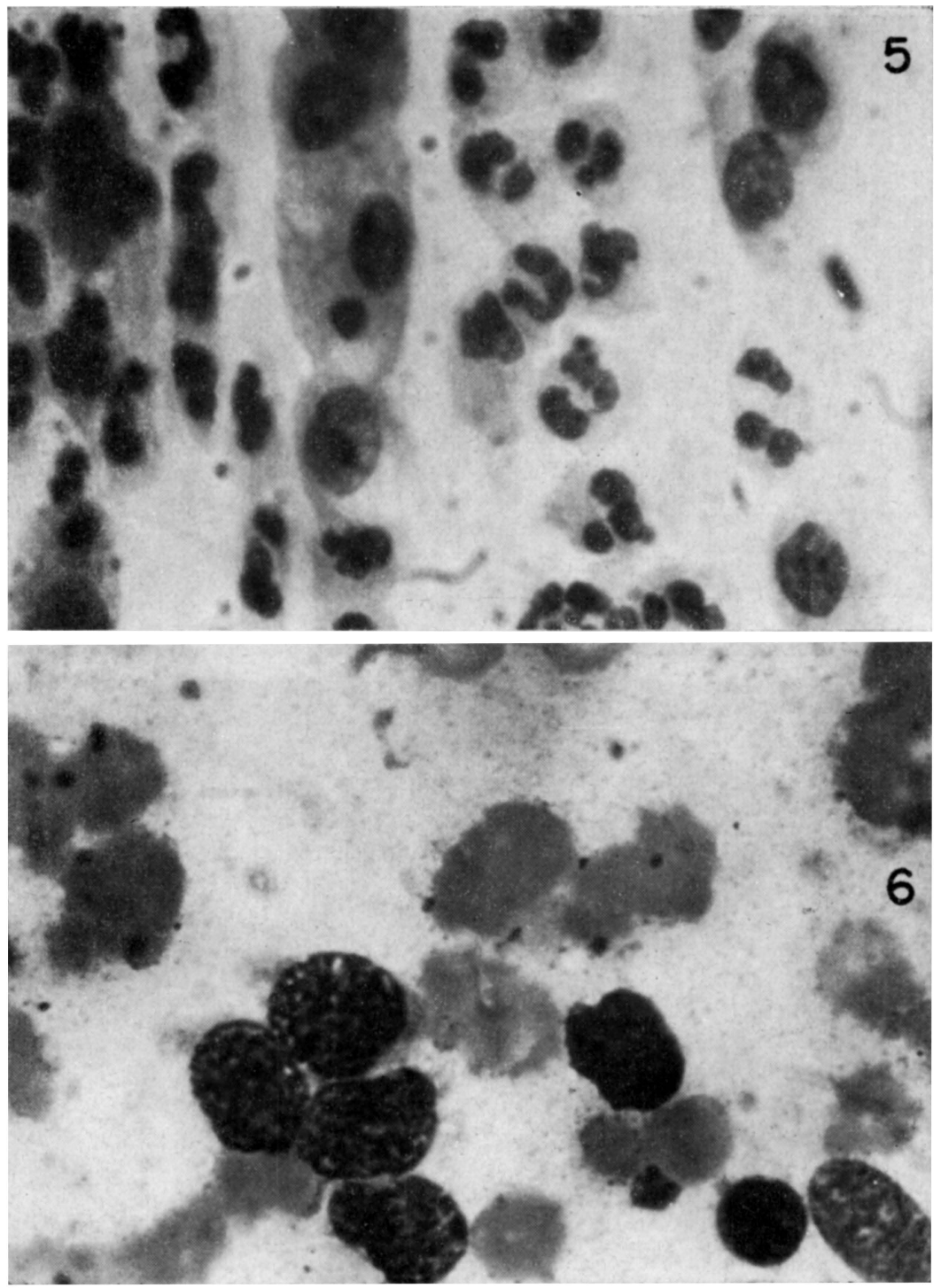

Fig. 5 - Esfregaço vaginal mostrando grupos de células atípicas, com citoplasma vacuolado (Papanicolau, $1000 \times)$.

Fig. 6 - Células neoplásicas no exame colpocitológico. Notar cromatina com grumos grosseiros (Papanicolau, $1000 \times)$. 
368 ARQ. NEURO-PSIQUIAT. (SÃO PAULO) VOL. 29, N.० 3, SETEMBRO, 1971
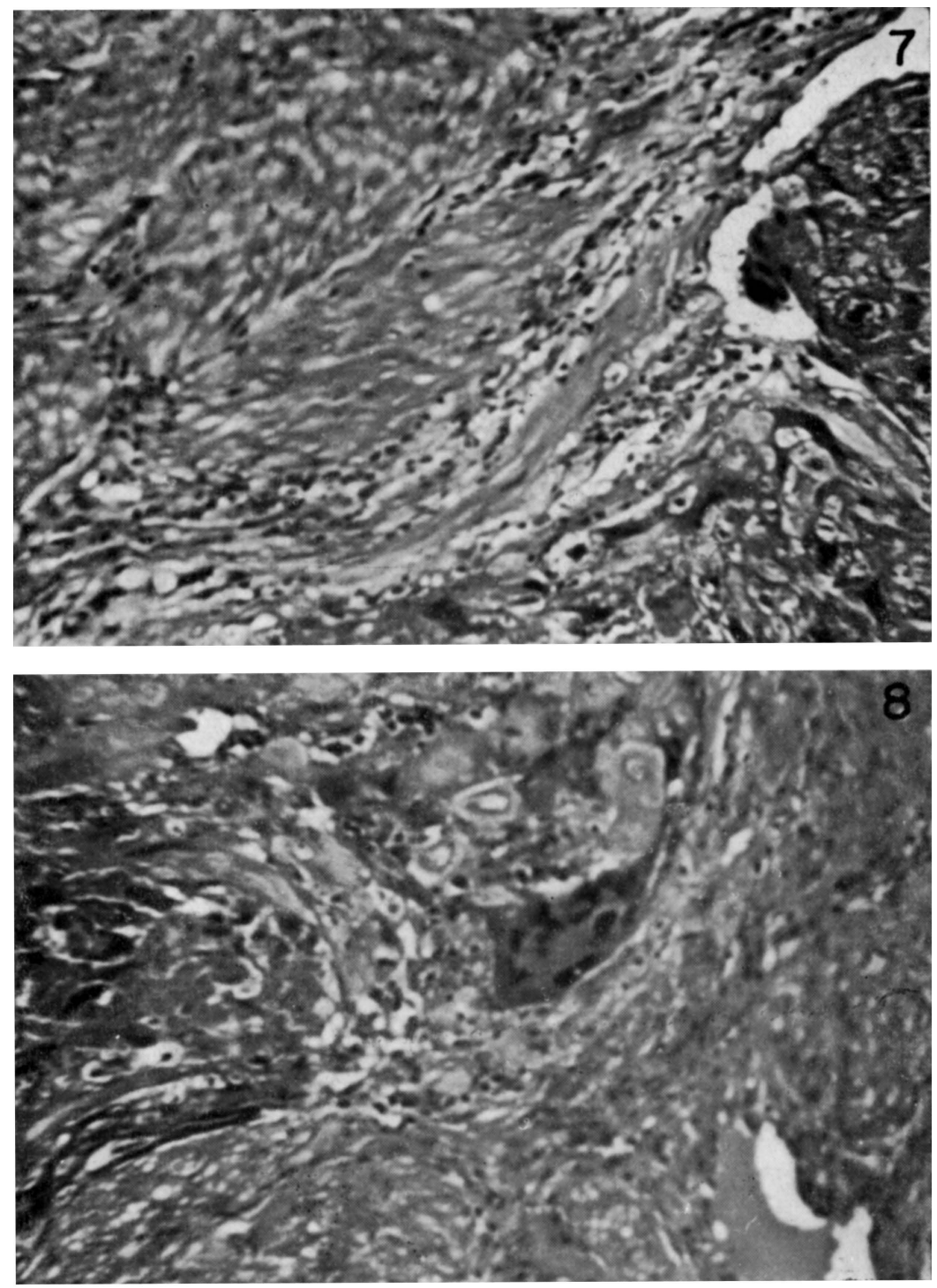

Fig. 7 - Massas de células neoplásicas infiltrando o miométrio $(H . E ., 450 \times)$.

Fig. 8-Células cito e sinciciotrofoblásticas penetrando na intimidade do tecido miometrial; infiltração inflamatória $(H . E ., 450 \times)$. 

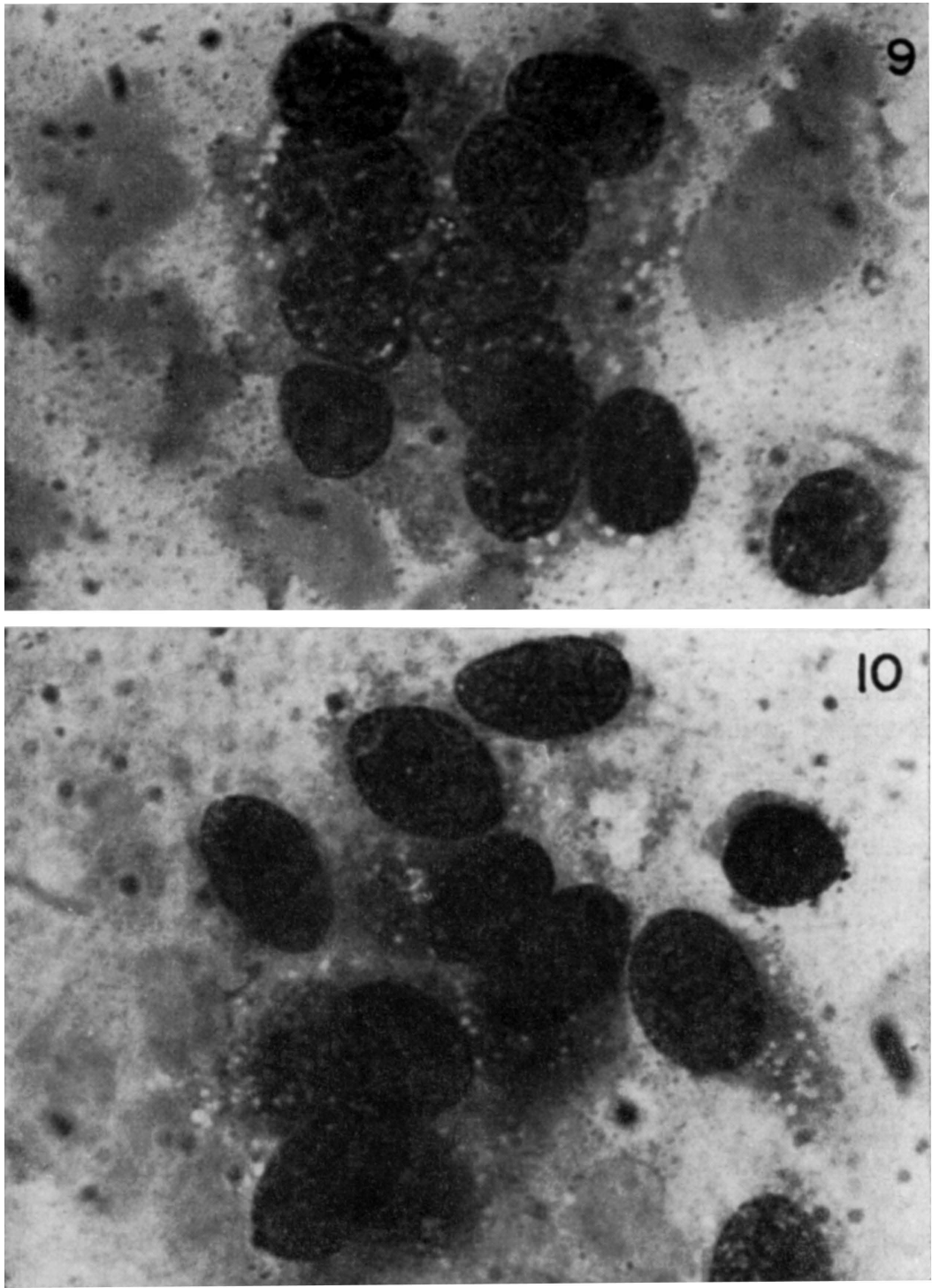

Fig. 9-Grupos de células citotrofoblásticas (células de Langhans) com hipercromatismo nuclear e vacuolização citoplasmática (Papanicolau, $1000 \times)$.

Fig. 10 - Células citotrofoblásticas em grupos; vacuolização citoplasmática $\boldsymbol{e}$ arranjo grosseiro da cromatina. Algumas células mostram macronucléolos (Papanicolau, $1000 \times)$. 
O coriocarcinoma é uma neoplasia maligna, resultante da degeneração dos elementos celulares normalmente presentes no trofoblasto e nas vilosidades coriais ${ }^{20}$, que apresenta grande tendência para a infiltração local com extensão para os tecidos vizinhos, além de grande capacidade para a formação de metástases. Juntamente com outros tumores trofoblásticos (mola hidatiforme, corioadenoma destruens ou mola invasiva), o coriocarcinoma se origina dos elementos trofoblásticos da placenta dos quais retém em potencial, a capacidade fisiológica e o comportamento biológico ${ }^{16}$.

As metástases cerebrais parecem ser freqüentes a julgar pelos dados estatísticos colhidos da literatura ${ }^{1,6,7,17,19}$. Contudo é difícil ter idéia exata da incidência das metástases cerebrais no coriocarcinoma. As falhas estatísticas são devidas principalmente à dificuldade em diagnosticar clìnicamente uma lesão desta natureza, bem como na difuculdade para examinar cérebros de indivíduos falecidos em muitos centros hospitalares. As lesões metastáticas podem ser únicas ou múltiplas e variar de dimensões. A localização da metástase pode variar bastante, podendo ocupar situação superficial ou penetrar profundamente na massa cerebral. No paciente estudado por Dockerty e Craig ${ }^{7}$ o tumor era subcortical estendendo-se posteriormente em direção ao lobo occipital. No caso descrito por Pereira e col. ${ }^{20}$ o tumor intracerebral determinava forte compressão do lobo temporal esquerdo, deslocando-o para fora, e invadia a protuberância.

O tratamento do coriocarcinoma tem sofrido acentuada mudança, em virtude dos brilhantes resultados obtidos com o uso de quimioterápicos, notadamente o metotrexato $12,13,14,22$, fazendo com que o prognóstico se torne menos sombrio, com vistas mesmo à possibilidade de cura. Contudo, a metástase cerebral precoce do coriocarcinoma é sempre de mau prognóstico.

Os autores descrevem um caso de coriocarcinoma cerebral metastático, removido cirùrgicamente, ocorrendo numa paciente de 19 anos e localizado na porção posterior do lobo frontal esquerdo. Um estudo retrospectivo da paciente revelou apenas discreta perda sangüínea pelos genitais, iniciada algumas semanas após o delivramento de uma criança normal, ocorrido quatro meses antes. O exame colpocitológico da secreção cérvico-vaginal revelou uma classe $\mathrm{V}$ de Papanicolau (positivo para células neoplásicas malignas), tendo ulteriormente a paciente sido submetida à histerectomia total com anexectomia bilateral. Um pequeno tumor de aspecto hemorrágico-necrótico foi encontrado na cavidade uterina, tendo o exame histológico mostrado tratar-se de coriocarcinoma. Os autores tecem considerações clínico-patológicas sôbre o coriocarcinoma. com escecial referência às lesões metastáticas cerebrais. 


\section{Metastatic cerebral chorioncarcinoma simulating a primary brain tumor}

A case of a metastatic cerebral chorioncarcinoma in the posterior aspect of the left frontal lobe of a 19-years-old woman is reported. After neurosurgical excision of the brain tumor a retrospective study of the patient was carried out as far as the gynecologic complaints were concerned. The patient complained only of little blood loss through the genitals started some weeks after the birth of a normal child four months ago. A smear vaginal preparation showed the presence of atypical epithelial cells (class $\mathrm{V}$ of Papanicolau). A total hysterectomy associated with bilateral anexectomy was performed. A small hemorrhagic tumor was detected within the uterus and a histological examination showed a chorioncarcinoma invading the miometrium. The clinico-pathological aspects of the chorioncarcinoma with special emphasis to cerebral metastases are discussed.

\section{R E F E E N C I A S}

1. ACOSTA-SISON, H. - The relative frequency of various anatomic sites as the point of first metastasis in 32 cases of chorionepithelioma. Am. J. Obst. \& Gynec, 75:1149, 1958.

2. AHUMADA, J. C.; CHEVAliER, H. M. \& SAMMARTiNo, R. - Corioncarcinomas. In El Cancer Ginecológico. Ahumada et al., editores. El Ateneo, Madrid, 1953.

3. AINSWORTH, H. W. \& GRESHAM, G. A. - Primary chorioncarcinoma of the urinary bladder in the male. J. Path. Bact. 79:185, 1960.

4. BARZILAI, G. - Atlas of Ovarian Tumors. Grune \& Stratton, New York, 1943.

5. BEACHAM, D. W. \& BEACHAM, W. D. - Synopsis of Gynecology. C. V. Mosby Co, New York, 1967.

6. DIXON, F. J. \& MOORE, R. A. - Testicular tumors. A clinico-pathological study. Cancer 6:427, 1953.

7. DOCKERTY, w. C. \& MCK CRAIG, W. - Chorionepithelioma. An unnusual case in which cerebral metatasis occurred four years after hysterectomy. Amer. J. Obst. \& Gynec. 44:497, 1942.

8. DougaL, D. - Chorionepithelioma of the ovary. J. Obst. Gynaec. Brit. Emp. 31:387, 1924.

9. FORTHER, H. C. \& OWEN, S. E. - Chorionepithelioma in the male. Amer. J. Cancer 25:89, 1935.

10. FRIEDMAN, N. B. \& MOORE, R. A. - Tumors of testis: a report on 992 cases. Milit. Surg. 99:573, 1946.

11. HERTIG, H. T. \& MANSELL, H. - Tumors of the Female Sex Organs. Part. I Hydatiform mole and Chorioncarcinoma. Atias of Tumor Pathology, section IX, fascicle 33. Armed Forces Institute of Pathology, Washington D.C., 1956.

12. HERTZ, H.; ROSS, G. T. \& LIPSETT, M. B. - Chemotherapy in women with trophoblastic disease: chorioncarcinoma, chorioadenoma destruens and complicated hydatiform mole. Ann. N. Y. Acad. Sci. 114:881, 1964.

13. LAMBE, J.; MORTON, D. G. \& BYRON, R. C. - Methotrexate therapy of chorioncarcinoma and allied tumors. Amer. J. Obst. \& Gynec. 90:317, 1964.

14. LI, M. C.; HERTZ, R. \& SPENCER, D. B. - Effect of methotrexate therapy upon chorioncarcinoma and chorionadenoma. Proc. Soc. Exper. Biol. Med. 93: $361,1956$. 
15. MARRUBINI, G. - Primary chorionepithelioma of the ovary: report of two cases. Acta obstet. gynec. scand. 28:251, 1948-1949.

16. MOORE, R. M. - Hormonal aspects and diagnosis of trophoblastic tumors. In New Concepts in Gynecological Oncology. Lewin-Wentz-Jaffe, editors, F. A. Davis Co., New York, 1966.

17. NOVAK, E. \& SEAH, C. S. - Chorioncarcinoma of the uterus. Study of 74 cases from the Mathieu Memorial Chorionepithelioma Registry. Amer. J. Obstet. \& Gynec, 67:933, 1954.

18. OLIVER, H. M. \& HORNE, E. O. - Primary teratomatous chorionepithelioma of the ovary: report of a case. New England J. Med. 239:14, 1948.

19. PARK, W. W. \& LEES, J. C. - Chorioncarcinoma. A general review with an analysis of 516 cases. Arch. Path. 49:104, 1950.

20. PEREIRA, W. C.; MATTOSINHO-FRANÇA, L. C.; TENUTO, R. A. \& EPPS, D. - Teratocorioepiteliomas: relato de um intracraniano e outro ovariano. Maternidade e Infância (São Paulo) 25:117, 1966.

21. PUGSLEY, W. S. \& CARLETON, R. L. - Germinal nature of teratoid tumors of the thymus. Arch. Path. 56:341, 1953.

22. VOGLER, W. R.; HUGULEY, C. M. \& KERR, W. - Toxicity and anti-tumor effect of divided doses of methotrexate. Intern. J. Med. 115:285, 1965.

Hospital de Cancer Napoleão Laureano - João Pessôa - Paraiba - Brasil. 